\title{
PAPER
}

\section{Longitudinal study of cognitive dysfunction in multiple sclerosis: neuropsychological, neuroradiological, and neurophysiological findings}

\author{
M R Piras, I Magnano, E D G Canu, K S Paulus, W M Satta, A Soddu, M Conti, \\ A Achene, G Solinas, I Aiello
}

See end of article for authors' affiliations

Correspondence to: Dr I Magnano, Institute of Clinical Neurology, University of Sassari, Viale San Pietro 10,07100 Sassari, Italy: imagnano@uniss.it

Received 9 July 2002 Accepted in final revised form 3 March 2003

\begin{abstract}
Objective: (1) To assess cognitive function and cerebral magnetic resonance imaging (MRI) involvement in relapsing-remitting multiple sclerosis; (2) to monitor disease evolution, cognitive dysfunction, and cerebral lesion burden over time (mean 8.5 year follow up period); (3) to study the relation between clinical, neuropsychological, and MRI data. On follow up assessment, visual and auditory oddball event related potentials (ERPs) were recorded as psychophysiological evaluation of cognitive status. Correlations between neuropsychological, MRI, and ERP data were also analysed.

Methods: Neuropsychological study assessed verbal and non-verbal IQ, deterioration index (DI) from WAIS subtests, conceptual reasoning, attention, verbal and visuospatial short-term and long term memory. MRI assessment detected presence of demyelinating lesions by using a semiquantitative method as well as cortical and subcortical atrophy over time.

Results: Attention, short-term and long term visuospatial memory were mildly impaired at baseline and remained unaltered longitudinally. At retesting a significant worsening of verbal long term memory $(p=0.023), D I$ presence $(p=0.041)$ and the increase of supratentorial and subtentorial MRI lesions load $(p=0.001)$ emerged. Expanded disability status scale score correlated significantly with total lesion burden at both evaluations ( $p=0.043$ and $p=0.024$ respectively). Temporal, occipital, and frontal horn lesions as well as cortical atrophy correlated significantly with attention and memory tests at baseline. Follow up assessment revealed significant correlation between cortical atrophy and attention as well as visuospatial short-term memory; spatial long term memory correlated significantly with lesions in body of lateral ventricle and frontal lobe. ERP study showed P300 latency abnormalities in 75\% of patients, involving specifically more visual P300 (58.4\% of cases) than auditory wave (41.6\%). Visual P300 latency and amplitude correlated significantly with DI and auditory P300 latency with frontal horn and brain stem lesions.

Conclusions: These findings revealed mild cognitive impairment in MS patients particularly consistent with slowing information processing over time. Increased MRI lesions do not correlate with the clinical course of the disease and cognitive deficit evolution. Thus, cognitive dysfunction could be related to disease peculiarity and not to the time course. Correlations between P300, neuropsychological, and MRI findings provide further information about ERP application to examine cognitive impairment in MS and probably to investigate their neural origin.
\end{abstract}

Abbreviations: MS, multiple sclerosis; MRI, magnetic resonance imaging; EDSS, expanded disability status scale; RR MS, relapsing-remitting MS; CPMS, chronic progressive MS; SP MS, secondary progressive MS; WAIS, Wechsler adult intelligence scale; DI, deterioration index; ERP, event related potential 
significantly. ${ }^{18}$ During a three year follow up study, Patti et a $l^{19}$ found only a significant increased Kurtzke's expanded disability status scale score (EDSS) ${ }^{20}$ in SP MS patients. Feinstein et al followed up patients with clinically isolated lesions (CIL) for a mean 4.5 years investigating cognitive, psychiatric, and MRI features. ${ }^{21}$ A significant decline in visual memory performance emerged on retesting. Zivadinov et al demonstrated that cognitive deterioration relies more on the development of brain parenchymal volume atrophy than on the extent of burden of disease in the brain during a two year follow up investigation in RR MS patients. ${ }^{22}$ Sperling et al investigated cognitive function and cerebral lesion burden by using regional magnetic resonance in MS patients. ${ }^{23}$ Demyelinating lesions in frontal and parietal white matter were strongly associated with performances requiring sustained complex attention and working verbal memory and this relation was consistent over a four year follow up period.

Cognitive function in MS patients has been studied also by psychophysiological approach, recording event related potentials (ERPs) using an oddball paradigm. The P300 wave, reflecting the timing and processing of mental activity during a simple task, has been widely used in MS. Correlations between P300 latency and/or amplitude and disease duration, neuropsychological testing and MRI are still controversial. ${ }^{24-29}$

The aims of this study were to assess neuropsychological and MRI profile in clinically definite RR MS patients and to investigate cognitive dysfunction and MRI lesion burden evolution during a mean 8.5 year follow up.

In addition, visual and auditory ERPs (P300) were recorded as psychophysiological evaluation of cognitive impairment at follow up retesting.

\section{METHODS}

Twelve MS patients ( 10 women and 2 men) underwent the follow up study. The patients met the Poser criteria ${ }^{30}$ for clinically definite RR MS. Physical disability was rated using Kurtzke's EDSS score. ${ }^{20}$ Patients gave informed consent before being included in the study. The patients were in agreement with inclusion criteria such as EDSS score $\leqslant 4$, clinical remission of the disease for three months, free of corticosteroid treatment for at least three months.

Exclusion criteria were the presence of other neurological or psychiatric illness, presence of severe depression, neuroleptic, antidepressant, or antiepileptic drugs.

Presence or absence of depression was evaluated through the Hamilton Rating Scale. ${ }^{31}$ Minor depression was present in three patients at entry and in four patients at the follow up assessment.

Handedness was assessed by Edinburgh Handedness Inventory ${ }^{32}$ : 11 patients were right handed and one patient was left handed.

Originally, 32 patients were recruited but after the 8.5 follow up period only 12 patients matched the inclusion or exclusion criteria. In fact, the disease course changed from RR to $\mathrm{CP}$ in 10 patients determining an EDSS score $>4$. In such a condition, they are unable to perform most of the neuropsychological testing because of the severe physical disability. Four patients performed neuropsychological study at baseline and follow up but did not undergo cerebral MRI after 8.5 years and six patients refused both neuropsychological and MRI follow up assessment at the second examination.

Neuropsychological, neuroradiological, and neurophysiological studies were performed in two or three consecutive days.

Table 1 summarises the patients' clinical characteristics.

\section{Neuropsychological assessment}

Neuropsychological evaluation was carried out to verify the following cognitive function by means of the related tests:

\begin{tabular}{|c|c|c|}
\hline \multirow[t]{2}{*}{$\begin{array}{l}\text { Sex (female/male) } \\
\text { EDSS } \\
\text { Mean (SD) age onset } \\
\text { Mean follow up (in months) } \\
\text { Handedness (right/left) }\end{array}$} & \multicolumn{2}{|c|}{$\begin{array}{l}10 / 2 \\
\leqslant 4 \\
26.8(7.3) \\
102 \text { (range } 79-125) \\
11 / 1\end{array}$} \\
\hline & Baseline & Follow up \\
\hline $\begin{array}{l}\text { Mean age (SD) } \\
\text { Disease duration (y) } \\
\text { EDSS } \\
\text { Education (y) } \\
\text { Depression (yes/no) }\end{array}$ & $\begin{array}{l}32.5(8.0) \\
7.7(6.3) \\
1.8(1.3) \\
9.2(4.1) \\
3 / 9\end{array}$ & $\begin{array}{l}40.6(8.2) \\
13.8(6.8) \\
2.5(0.9) \\
9.9(4.5) \\
4 / 8\end{array}$ \\
\hline
\end{tabular}

(1) General intellectual function. The Italian version of Wechsler adult intelligence scale, WAIS ${ }^{33}$ was administered and deterioration index (DI) was calculated according to the following Wechsler's formula:

$$
\frac{(\text { hold }- \text { don't hold }) \times 100}{\text { hold }}
$$

that uses age graded scores of five hold tests (information, vocabulary, picture completion, object assembly) and four do not hold tests (similarities, digit span, digit symbol, block design). ${ }^{34}$ The normal limit of each subtest was established considering the 5th percentile of control's performances as cut off point. The presence of DI is generally rated in percentage (\%) and positive percentage is indicative for "possible deterioration". Negative score (about $2 \%-3 \%$ ) are considered as absence of DI, but very high negative score may indicate the loss of some intellectual performances.

One patient (MAS74, female, aged 16) was assessed by the Wechsler intelligence scale for children revised, WISC- $\mathrm{R}^{35}$ and consequently the DI was not been calculated.

(2) Non-verbal reasoning: Raven's progressive coloured matrices. $^{36}$

(3) Visual attention: visual Search test. ${ }^{37}$

(4) Verbal short-term memory: digit span from WAIS verbal subtest. ${ }^{37}$

(5) Spatial short-term memory: corsi span. ${ }^{37}$

(6) Verbal long term memory: verbal supraspan-selective reminding test. ${ }^{38}$

(7) Spatial long term memory: learning spatial supraspan. ${ }^{37}$

(8) Language function: aphasia test. ${ }^{32}$

Results were compared with Italian published norms. ${ }^{33}$ 35-37

Individual test performance was considered abnormal when it was below the normal control mean. A grading system developed by Camp et al ${ }^{39}$ was applied to each patient test score, considering the number of standard deviations (SDs) below the control mean. In details, grade 0 was given when the patient scored at or above the control mean, grade 1 when the patient scored at or above 1 SD below the control mean, and grade 2 when the patient scored 2 or more SDs below the control mean. Patients were considered affected by impairment of a given cognitive domain when they scored grade 2 at least on one of the tests assessing the corresponding function.

The neuropsychological evaluation needed about two hours and it was performed in two sessions.

\section{MRI evaluation}

Baseline and follow up MRI scans were analysed by two neuroradiologists (MC and AA) blinded to the clinical, neuropsychological, and psychophysiological findings, providing an 
inter-rater reliability of 0.95 . To estimate presence and size of periventricular and discrete lesions, a semiquantitative method was used ${ }^{40}$ to score the total lesion burden and the presence of lesions in 15 different cerebral sites. Total number and size of plaques was counted in each site using a point scale ranging from 0 to 4 with higher numbers indicative of increasing severity. In fact, a score of 0 was related to the absence of demyelinating lesions, 1 was referred to lesions of maximum $0.5 \mathrm{~mm} ; 2$ to $0.6-10 \mathrm{~mm}$; 3 , lesion size $>10 \mathrm{~mm}$; the score 4 indicated lesions larger than score 3 . The scores from all 15 sites were then added to obtain the total score for each patient. The follow up assessment focused particularly to describe the increase in lesions. Furthermore, the presence of cortical and subcortical atrophy was also described.

Considering the long period of observation and the obvious upgrade of equipment, in some of the early scans (1987/1989, three patients) MRI images were $8 \mathrm{~mm}$ thick and the remaining slices consisted of $6 \mathrm{~mm}$ thickness. At follow up, all patients were submitted to brain MRI on IT Superconductive Unit (Shinadzu SMT-100x) at the Institute of Radiology of Sassari University. The routine examination consisted of spin echo (SE), conventional T1, proton density (DP), and T2 weighted acquisitions with axial and coronal slices of $5 \mathrm{~mm}$ thickness. Unfortunately, it was impossible for practical reasons to achieve precise re-positioning of MRI scans on these follow up examinations, considering also the different slice thickness. For these reasons, the MRI scan evaluation was performed in all cases by axial scan analysis and integrated with sagittal and coronal plane evaluation for a more detailed estimate of number and size of demyelinating lesions.

\section{ERP study}

Visual and auditory oddball ERPs were recorded at follow up using 30 scalp electrodes to elaborate topographical maps. The bandpass was 1-70 Hz, ground electrode placed in Fpz and reference at linked balanced earlobes. Eye artefacts were recorded by two supplementary channels to be eliminated during elaboration. Additional artefact rejection was kept on $\pm 40 \mu \mathrm{V}$. The visual oddball paradigm consisted of numeric stimuli (respectively, 1 as frequent stimulus and 0 as rare one) presented binocularly and randomly on a screen. In the auditory oddball paradigm, pitch tones $(1000 \mathrm{~Hz}$ frequent and $2000 \mathrm{~Hz}$ rare stimuli) were administered binaurally and randomly. Stimuli duration was $150 \mathrm{~ms}$, rise-fall time $30 \mathrm{~ms}$, and $1300 \pm 250 \mathrm{~ms}$ interstimulus period. The frequent/rare ratio was 4:1. An average of 20 rare stimuli was presented to the patients and each test was performed three times to verify the repeatability of the waves. The subjects, comfortably seated on a chair, were instructed to mentally count and press a button to respond to rare stimuli. In such a way, we monitored patients' attention level.

Each ERP component was identified according to its polarity, latency, and scalp distribution independently by two of the authors (IM and KSP) resulting in a low inter-rate variability between the two measurements. In the visual modality, the early components were distinguished in $\mathrm{Pl}$, reflecting the first positive deflection, $\mathrm{Nl}$ as the first negative deflection and $\mathrm{P} 2$ as the positive wave following the $\mathrm{Nl}$. The cognitive potentials were recognised only in response to the rare stimuli in averaged EEG and included the N200 and the P300, the first considered the most negativity between the P2 and the P300 and the second the most prominent positivity following the N200.

In the auditory modality, the early components were labelled N100 and P200 respectively verifying about $100 \mathrm{~ms}$ and $200 \mathrm{~ms}$ after the rare stimulus, reflecting the sensory processing. N200 was recorded after the P200 and before the late positive component called P300.

The amplitudes of ERP components were calculated with respect of the mean of EEG activity occurring during the 200 $\mathrm{ms}$ prestimulus baseline period scoring the peak to peak values. If the P300 amplitude was too small and the morphology of the trace was not well clear, the P300 wave was considered not identifiable. Results were compared with 15 sex, education, and age matched controls. A regression line was calculated for auditory and visual ERPs in controls and MS patients to evaluate the relation between age, latencies, and amplitude. Visual and auditory latency of N200 and P300 correlated significantly in controls (visual N200 $r=0.64 \mathrm{p}<0.03$, visual P300 $r=0.75 \mathrm{p}<0.02$; auditory N200 $r=0.72 \mathrm{p}<0.03$, visual P300 $r=0.81 \mathrm{p}<0.01$ ). In contrast, both N200 and P300 amplitude did not show significant correlation with age. Such correlations were not evident for patient group. Abnormal latency of a component was defined as a value equal or greater than 2 SDs of age matched estimate calculated by means of the linear regression analysis.

\section{Statistical analysis}

Neuropsychological and neuroradiological data were statistically computed to investigate correlations at baseline and follow up by using paired $t$ tests or Wilcoxon matched pairs signed rank tests depending on whether the data distribution was considered to be normal or not.

Correlations between variables at baseline and follow up were assessed with the Spearman rank correlation coefficients.

The software used for the analysis of the data was the statistical package for the social sciences (SPSS) for Windows version 10.0 .

\section{RESULTS}

\section{Neuropsychological findings}

Before administering the psychometric test battery, patients' language function (oral and written comprehension and production) were assessed and none of them showed abnormalities at both evaluations.

\begin{tabular}{|c|c|c|c|c|c|c|}
\hline & \multicolumn{2}{|l|}{ Grade 0} & \multicolumn{2}{|l|}{ Grade 1} & \multicolumn{2}{|l|}{ Grade 2} \\
\hline & Baseline & Follow up & Baseline & Follow up & Baseline & Follow up \\
\hline WAIS full scale IQ & 8 & 7 & 1 & 3 & 3 & 2 \\
\hline Raven progressive matrices & 9 & 9 & 2 & 3 & 1 & 0 \\
\hline Visual search & 5 & 7 & 6 & 3 & 1 & 2 \\
\hline Digit span-WAIS & 10 & 9 & 1 & 3 & 1 & 0 \\
\hline Corsi span & 5 & 8 & 6 & 3 & 1 & 1 \\
\hline Verbal supraspan & 9 & 3 & 1 & 7 & 2 & 2 \\
\hline Learning spatial supraspan & 8 & 5 & 2 & 5 & 2 & 2 \\
\hline
\end{tabular}

WAIS, Wechsler Adult Intelligence Scale. Each psychometric test was scored according to the grade system by Camp et $a^{\beta 9}$ developed on the numbers of SDs. Grade 0, score at normal value. Grade 1, score $\geqslant 1$ SD below normal value. Grade 2, score $\geqslant 2$ SDs below normal value. 
Table 3 Mean scores of MS patients at baseline and follow up neuropsychological testing

\begin{tabular}{llll}
\hline Neuropsychological battery & Baseline & Follow up & $\mathrm{p}$ \\
\hline WAIS full scale IQ & 92.1 & 91.3 & $\mathrm{NS}$ \\
Raven progressive matrices & 27.7 & 28.3 & $\mathrm{NS}$ \\
Deterioration index (DI) & 5.3 & 11.8 & $\mathbf{0 . 0 4 1}$ \\
Visual search & 52.6 & 48.2 & $\mathrm{NS}$ \\
Digit span & 6.2 & 6.4 & $\mathrm{NS}$ \\
Corsi span & 5.2 & 5.0 & $\mathrm{NS}$ \\
Verbal supraspan & 8.6 & 7.8 & $\mathbf{0 . 0 2 3}$ \\
Visual spatial supraspan & 7.0 & 22.5 & NS \\
\hline
\end{tabular}

Comparison between the baseline and the follow up was performed by paired $t$ test. Significant $p$ value $<0.05$ in bold letters; NS, not significant value, $p>0.05$. Note the different version of visual spatial supraspan test at initial (only retained items) and final assessment (by Spinnler and Tognoni $\left.{ }^{37}\right)$.

Table 4 Mean scores (SD) of WAIS subtests at baseline and follow up

\begin{tabular}{|c|c|c|c|}
\hline & Baseline & Follow up & $\mathrm{p}$ \\
\hline \multicolumn{4}{|l|}{ Verbal subtests } \\
\hline Information * & $8.9(3.1)$ & $9.5(2.4)$ & NS \\
\hline Comprehension & $9.9(3.0)$ & $7.8(3.9)$ & NS \\
\hline Arithmetic & $8.6(2.6)$ & $7.3(2.0)$ & NS \\
\hline Similarities $†$ & $10.3(2.5)$ & $9.6(4.4)$ & NS \\
\hline Digit spant & $8.1(1.6)$ & $7.3(3.1)$ & NS \\
\hline Vocabulary* & $8.9(2.4)$ & 7.9 (3.7) & NS \\
\hline \multicolumn{4}{|l|}{ Performance subtests } \\
\hline Digit symbolt & $6.2(2.5)$ & 6.7 (1.9) & NS \\
\hline Picture completion* & $9.0(1.8)$ & $8.9(2.0)$ & NS \\
\hline Block designt & $8.5(2.3)$ & $8.1(1.6)$ & NS \\
\hline Picture arrangement & $8.3(2.2)$ & $8.8(3.9)$ & NS \\
\hline Object assembly* & $7.5(2.7)$ & $8.2(2.2)$ & NS \\
\hline
\end{tabular}

*Hold tests and †do not hold tests of WAIS considered for deterioration index calculation. Comparison between the baseline and the follow up was performed by paired $t$ test; NS, not significan value $p>0.05$.

Table 2 shows baseline and follow up neuropsychological performances of patients for each test according to the above mentioned grading system. Mean neuropsychological test scores at baseline and follow up are summarised in table 3. A statistically significant decline was present only on the DI $(p=0.041)$ and the verbal long term memory test $(p=0.023)$.

WAIS subtests: deterioration index

DI presence was detected in six patients $(50 \%)$ at baseline and in seven patients $(58.4 \%)$ at the follow up examination. Such
Table 5 MRI study: scores of significant increased burden lesion

\begin{tabular}{llll}
\hline & Baseline & Follow up & $\mathrm{p}$ \\
\hline Body of lateral ventricle & 5.8 & 12.3 & 0.010 \\
Frontal horn & 0.8 & 2.3 & 0.027 \\
Temporal horn & 2.1 & 2.6 & 0.046 \\
Trigone & 6.5 & 16.2 & 0.009 \\
Internal capsula & $00: 06$ & $01: 03$ & 0.025 \\
Frontal lobe & 5.5 & 10.8 & 0.004 \\
Parietal lobe & 1.1 & 2.9 & 0.004 \\
Temporal lobe & 0.8 & 2.3 & 0.037 \\
Cerebellum & 0.8 & 2.2 & 0.032 \\
& & & \\
Total burden lesions & 36.8 & 74.1 & 0.001 \\
\hline
\end{tabular}

Presence and size of lesions were scored by Ormerod method. ${ }^{40}$ Comparison between the baseline and the follow up was performed by Wilcoxon matched pairs test. Significant $p$ value $<0.05$.

data were related to 11 patients (leaving out MAS74 patient) because of different methods used between the baseline and follow up testing as mentioned in Methods. The mean values did not show any significant changes as shown in table 4 .

At the second assessment, five patients displayed very negative values of DI (ranging from $-2.7 \%$ to $-60.90 \%$ ). To explain such negative scores, we evaluated the performance of each WAIS subtest. So, we noted that when the 5 th percentile of controls' performances was used as a cut off, five patients (41.6\%) failed at least one of those subtests related to the abstract/conceptual reasoning skills as arithmetic, comprehension, and vocabulary.

\section{RMN study}

Twelve RR MS patients underwent brain MRI. According to Ormerod criteria, we scored a total lesion burden and lesion load in each of periventricular and discrete lesions in agreement to a score ranging from 0 (absence of demyelination) to 4 (severe lesions load). Total and specific MRI score index of cerebral areas are reported in table 5. Figure 1 shows the increase of lesion burden.

\section{ERP recordings}

The early components of visual ERPs (P1, N1, P2) showed a non-typical, robust morphology and a smaller amplitude compared to controls, but such differences failed to be significant. As for the auditory modality, the early components ( $\mathrm{Nl}$ and P2) were clearly identified in all patients.

Visual P300 wave was normal in five patients; in the remaining cases we observed three patients with delayed N200 and P300 latencies more than 2 SDs and four patients
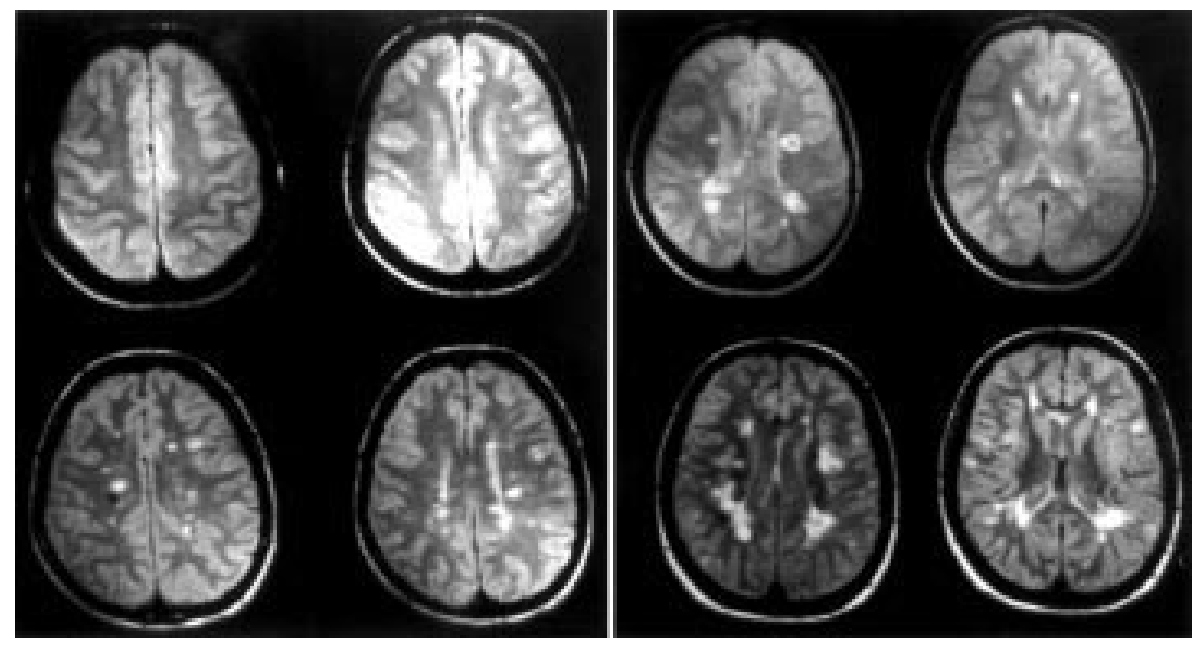

Figure 1 MRI scans of two patients showing the demyelinating lesions at baseline (top) and the increasing lesion burden at follow up examination (bottom). 

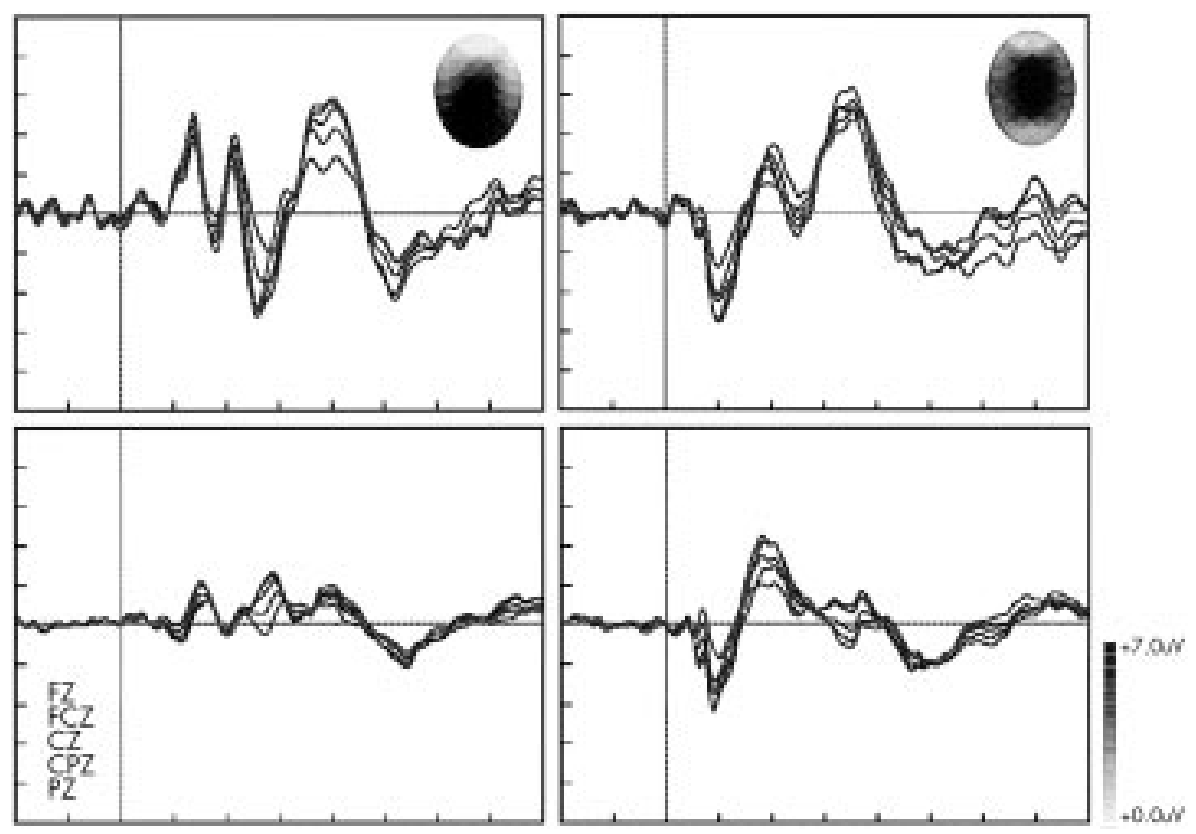

Figure 2 Grand averaged target visual (left) and auditory (right) oddball ERPs of controls (top) and MS patients (bottom). showing an unidentifiable N200 and P300. As a group, significant differences of $\mathrm{N} 200 \quad(p=0.03)$ and P300 $(p=0.04)$ latencies were found in MS patients compared with the controls (patients: N200 279,1 (28.2) ms, P300 376.2 (25.4) ms; controls: N200 248.6 (15.6) ms, P300 353.1 (15.2) ms).

Auditory ERP recordings revealed normal morphology, latency, and amplitude in seven patients. Four patients showed an increased latency of N200 and P300 (more than 2 SDs) and one patient had no N200 and P300 wave that could be clearly identified. Auditory N200 and P300 latencies of MS group were significantly prolonged $(\mathrm{p}=0.02$ and $\mathrm{p}=0.04$ respectively) compared with the controls (patients: N200 240,1 (26.3) ms, P300 354.7 (21.1) ms; controls: N200 217.1 (14.2) ms, P300 328.4 (25.6) ms)).

Visual and auditory P300 amplitudes were significantly reduced in patients compared with controls $(\mathrm{p}=0.041$ and $\mathrm{p}=0.021$ respectively). Figure 2 illustrates the visual and auditory ERP waves and their topographical distribution.

In our MS patient group, considering the delayed latency, the smaller amplitude and the cases with a not clearly identifiable P300, ERP abnormalities occurred in $75 \%$ of patients with the visual P300 more impaired ( 7 of $1258.4 \%$ of cases) than auditory wave ( 5 of $12 \quad 41.6 \%$ ). Topographical distribution, assessed by brain mapping representation, did not seem significantly changed with respect to control group.

\section{Statistical analysis of EDSS, neuropsychological, MRI, and ERP data}

Both initial and follow up correlations between neuropsychological tests, EDSS score, total and specific area MRI score index were analysed.

At baseline, significant correlations were found between the following variables: EDSS and total MRI score $(r=0.59$; $\mathrm{p}=0.043)$, WAIS-digit span test $(r=0.58 ; \mathrm{p}=0.046)$, learning spatial supraspan test $(r=0.60 ; \mathrm{p}=0.039)$, cerebellum $(r=0.67$; $\mathrm{p}=0.016)$, cortical atrophy $(r=0.59 ; \mathrm{p}=0.044)$. Because of these correlations, further analyses were performed to remove the possible effect of EDSS score on neuropsychological performances. Physical disability effects were assessed with an analysis of covariance with EDSS score as the covariate and no significant correlations were found.

Visual search test correlated significantly with temporal horn $(r=0.62 ; \mathrm{p}=0.045)$, occipital horn $(r=0.72 ; \mathrm{p}=0.007)$, frontal horn $(r=0.62 ; \mathrm{p}=0.029)$, and cortical atrophy $(r=0.58$; $\mathrm{p}=0.046)$.
Verbal short-term memory (WAIS digit span) was significantly correlated with occipital horn $(r=0.60 ; \mathrm{p}=0.037)$ and presence of atrophy $(r=0.99 ; \mathrm{p}=0.001)$. Verbal (verbal supraspan) and spatial (learning spatial supraspan test) long term memory test yielded significance respectively with parietal lobe $(r=0.57 ; \mathrm{p}=0.049)$ and occipital horn $(r=0.66$; $\mathrm{p}=0.019)$ and with internal capsula lesions $(r=0.78 ; \mathrm{p}=0.002)$ and cortical atrophy $(r=0.69 ; \mathrm{p}=0.01)$.

At follow up statistical correlations were significant between the following variables: EDSS and total MRI score $(r=0.64 ; \mathrm{p}=0.024)$, frontal horn $(r=0.72 ; \mathrm{p}=0.007)$, fourth ventricle $(r=0.76 ; \mathrm{p}=0.003)$, cerebellum $(r=0.62 ; \mathrm{p}=0.029)$. Performances on Raven test showed significant correlations with brain stem lesions $(r=-0.65 ; \mathrm{p}=0.020)$ and cortical atrophy $(r=0.59 ; \mathrm{p}=0.042)$. Visual attention correlated significantly with cortical atrophy $(r=0.62 ; \mathrm{p}=0.031)$ as well as visuospatial short-term memory $(r=0.86 ; \mathrm{p}=0.001)$. Learning spatial supraspan test presented significant correlations with lesions in body of lateral ventricle $(r=0.58 ; \mathrm{p}=0.044)$ and frontal lobe plaques $(r=0.58 ; \mathrm{p}=0.044)$.

Statistical analysis of neuropsychological data and ERP findings revealed significant correlations between degree of DI and visual N200 latency $(r=-0.59 ; \mathrm{p}=0.041), \mathrm{P} 300$ latency and amplitude (respectively, $r=-0.74 ; \mathrm{p}=0.005$ and $r=-0.62$; $\mathrm{p}=0.029)$ besides of auditory P300 latency and frontal horn $(r=-0.64 ; \mathrm{p}=0.024)$ and brain stem $(r=-0.80 ; \mathrm{p}=0.001)$ lesions.

\section{DISCUSSION}

This longitudinal study aimed at evaluating cognitive impairment in RR MS patients in relation to the clinical course of the disease, the increased cerebral lesion load, and brain atrophy over a mean 8.5 year follow up. During the whole observation period, the EDSS score was inferior or equal to 4 as to evaluating subjects without neurological disability. In fact, a very careful physical and neurological examination was conducted to assess the integrity of fine motor functioning, muscular force and praxic skills every six months over the whole follow up. In such a way the neuropsychological testing, requiring a good motor and speed performance and a fine visual acuity, was not influenced by physical disability. However, the relation between neuropsychological findings and EDSS score is still debated in literature and most papers report that MS patients could suffered from cognitive impairment even in less 
disabling form of the disease. ${ }^{2}{ }^{41}{ }^{42}$ We excluded any possible effect of EDSS score (that is physical handicap) on neuropsychological performances by means of analysis of covariance, with EDSS score as covariate.

At baseline evaluation, a reliable method to assess the global cognitive status was the full WAIS battery administration and the DI calculation. Also specific attention and memory tests were selected to complete the neuropsychological assessment. On retesting, a significant worsening of DI and long term verbal memory emerged. DI presence indicates a slowing of speed elaboration and attention with poor performances of immediate memory tests and abstract reasoning tests (digit span, digit symbol, block design, similarities). Recently, the validity of DI in identifying a real intellectual deterioration has been questioned but at retesting we considered such parameter because of methodological rigour and because of its significant changes.

The global IQ seemed not to be impaired on baseline and over time; consequently we analysed the patients' performances of each WAIS subtest to better clarify the individual differences. Overall, most verbal performance WAIS subtests (comprehension, arithmetic, vocabulary, and digit span) showed a mild worsening in an increased number of patients after the follow up period. This may be indicative of impairments in information processing activation to recall and organise the right response, as to word meaning, and problem solving.

Among our patients, long term verbal memory was significantly compromised after the 8.5 follow up period whereas the remaining neuropsychological domains remained unchanged. In our study, poor performances on Verbal Supraspan Test were scored by using the delayed recall score of the Selective Reminding Test. ${ }^{38}$ Such findings could account for an impairment of intentional retrieval in agreement with previous papers. ${ }^{15111639}$ In contrast with our findings, Faglioni et al carried out a cross sectional study in RR MS patients demonstrating deficits on encoding and automatic retrieval by using only unrelated items and paired associated items of the Selective Reminding Test. ${ }^{43}$ The different methodology and especially the long period of observation led us to conclude that slowing information processing could be also responsible for intentional retrieval deficit that becomes evident over time.

However, the declining cognitive functions did not seem to interfere in any particular way either in the daily life impact of the patients or in their working activity. In fact, our patients have achieved their own purpose in life studying, getting married, giving birth, and not developing mood disturbances or severe depression.

Our results support the hypothesis that cognitive impairment in RR MS patients occurs with a peculiar pattern of deficit that is called in literature mild cognitive impairment. Moreover, in agreement with the findings of other authors, ${ }^{16} 171921{ }^{44}$ we confirm that RR MS may be considered a peculiar clinical entity with benign course and low physical disability. Overall, our cohort of RR MS patients since initial clinical assessments (performed every six months over the duration of the study) displayed a stable clinical condition. In our opinion, clinical parameters of our patients, such as low EDSS score, RR disease course, one or two annual relapse over the follow up period, could be considered possible predictive factors of a distinctive cognitive decline. In fact, in our patient group as a whole, neuropsychological performances were initially impaired and remained more or less stable after 8.5 years. As for RR MS, the benign course of the disease would therefore suggest some evidence that such a mild cognitive impairment may occur in this form of the disease. Unfortunately, the comparatively small number of patients examined in this study precluded the use of statistical analysis to demonstrate the possible predictors of neuropsychological performances.

Cerebral MRI lesion burden dramatically increased in size and numbers of lesions at the follow up examination but not the same was evident regarding the clinical course of the disease and cognitive performances. Correlations between MRI total load and EDSS score on both baseline and follow up studies were found, but the total MRI lesion index did not seem a good factor for predicting the clinical course of the disease and the cognitive impairment. Partially, this finding may be due to the EDSS score $\leqslant 4$ as inclusion criterion for the enrolment in the study. It is known that controversial findings on the correlations between physical disability, EDSS score and total MRI brain load could be explained by different methodologies in evaluating MRI images and by varying disease course and duration. ${ }^{22}{ }^{42}{ }^{45-50}$ Also, in long term MRI follow up studies the comparison between images has to take into account the different thickness of slices. ${ }^{21}$ In our study the comparison was performed because only few baseline MRI images (three patients) presented an $8 \mathrm{~mm}$ thickness and the remaining slices had $6 \mathrm{~mm}$ of thickness. At the second evaluation, all MRI scans were of $5 \mathrm{~mm}$ thick permitting a feasible comparison. Body of lateral ventricle, trigone, frontal and temporal lobe lesions, occipital, temporal and frontal horn, brain stem were the most involved regions in demyelinating process; in our group, frontal horn and frontal lobe lesions correlated significantly with memory tests and ERP findings. Such lesion locations could be on the basis of the specific cognitive deficit, as reported by many authors. ${ }^{14}{ }^{48-54}$ Most of the significant correlations between neuropsychological and RMN data displayed at baseline were not evident at retesting. This would suggest that the cognitive impairment course appears less ingravescent than white matter brain demyelination, which nevertheless does not seem to influence the instrumental function of wide cognitive domains. Cognitive dysfunction, then, could be related to disease peculiarity occurring on a patient specific basis and not to the time course. Even though there was an increasing lesion load, the stable cognitive impairment could be explained by small lesions not detected by MRI scans. Such lesions could play a crucial part more than large lesions in a less critical area of brain for cognitive functioning. In fact, recent special neuroimaging techniques permit detection of microscopic cerebral lesions not seen on conventional methodology and such small lesions could account for specific cognitive deficits. ${ }^{55} 56$

Neurophysiological study was carried out to obtain an online measure of cognitive status in our MS patients and in particular to evaluate the late cognitive components N200 and $\mathrm{P} 300$. The N200 seems to indicate an early cognitive elaboration concerning subject's attention orientation. The P300 represents the closing elaboration of stimuli discrimination. ERP findings revealed a more impairment of visual P300 than auditory one. The sensory processing of early visual components of ERPs in the patients displayed lower amplitude compared with controls, but it was not significant. Furthermore, all patients suffered from optic neuritis during the time of observation but the early components evoked by visual stimuli showed a not significant trend of reduction amplitude and the subsequent late cognitive potential were normal or not in our sample independently by these data. Our findings could be explained by the different nervous pathway involved in visual and auditory stimuli processing as well as to the different generators implicated in processing these dissimilar kinds of stimuli. ${ }^{57-59}$ However, the widespread brain lesion burden is undoubtedly the phenomenon underlying these results. In fact, most MS lesions were seen on the body of lateral ventricle, trigone, frontal and temporal lobe lesions, occipital, temporal and frontal horn, brain stem that are known as cerebral regions of specific cognitive functions as memory, attention, and executive function. ${ }^{6061}$ According to literature data, our findings confirm that frontal horn and brain stem could be thought as critical areas of neural cognitive network involved in the neural origin of ERPs. Correlations between P300 and neuropsychological data and MRI findings provide further informations about the application of ERPs to examine cognitive impairment in MS as reported also by other 
authors. ${ }^{24-26}{ }^{62-64}$ Psychophysiological evaluation plays an important part in investigating mental disorders as well as known from literature; particularly ERPs represent a useful tool when cognitive status become impossible to study by means of conventional psychometric tests in course of diseases determining a severe physical disabilities. Thus, further psychophysiological studies are needed to monitor longitudinally cognitive deficits in MS patients, especially in CP MS patients who are not able to perform a full neuropsychological battery because of physical disability.

Despite the larger number of cross sectional investigations, only a few studies analysed neuropsychological findings and clinical informations longitudinally. Amato and colleagues followed up over 10 years a group of MS patients and a cognitive impairment emerged in an increasing proportion of patients during pathology progression from $26 \%$ to $56 \% .{ }^{17}$ At baseline, $74 \%$ of patients were cognitively unimpaired becoming $51 \%$ at the second retesting and $44 \%$ at the end of the study. However, it is worth mentioning that the RR MS group patients reduced from $88 \%$ to $58 \%$ and PP and SP MS patients reached $11 \%$ and $31 \%$ after 10 years being $12 \%$ and $0 \%$ at entry respectively. Very little combined neuropsychological and MRI follow up investigations are found in literature. These studies report data on comparatively short periods of observation ranging from one up to four years. ${ }^{18} 192223$ The longest follow up assessment was performed for 4.5 years by Feinstein in patients with clinically isolated lesions. ${ }^{21}$

To the best of our knowledge, this is the first study providing clinical, cognitive, and cerebral lesion burden data over about 8.5 years in RR MS patients. The very long period of observation is one of the strengths of our study although such long follow up studies have to take account the drop out rate determining a small sample of patients examined over time.

In conclusion, the pattern of cognitive impairment found in our MS patient group could be essentially indicative of reduced information processing speed causing intellectual slowing, attentional problems, impairment in abstract reasoning, problem solving, and memory dysfunction as distinctive features of "subcortical dementia". This condition could be attributable to the interruption of the neural connections among cortical associative areas as well as between cortical and subcortical structures as a consequence of demyelination and axonal degeneration. Thus, we hypothesise to name this peculiar MS cognitive pattern as subcortical dysconnection dementia in contrast with other clinical condition (Parkinson's disease, Huntington's disease, progressive supranuclear palsy) characterised by subcortical dementia, which is the consequence of a degenerative process of basal ganglia as crucial neuronal relay station for processing cognitive information.

\section{Authors' affiliations}

M R Piras, I Magnano, E D G Canu, K S Paulus, W M Satta, A Soddu, I Aiello, Institute of Clinical Neurology, University of Sassari, Sassari, Italy

M Conti, A Achene, Institute of Radiology, University of Sassari G Solinas, Laboratory of Epidemiology and Biostatistics, Institute of Hygiene and Preventive Medicine, University of Sassari

\section{REFERENCES}

1 Rao SM, Leo GJ, Bernardin L, et al. Cognitive dysfunction in multiple sclerosis. I. Frequency, patterns and prediction. Neurology 1991;41:685-91.

2 Peyser JM, Edwards KR, Poser CM, et al. Cognitive function in patients with multiple sclerosis. Arch Neurol 1980:37:577-9.

3 Kujala P. Portin R, Ruutiainem J. Memory deficits and early cognitive deterioration in MS. Acta Neurol Scand 1996;93:329-35.

4 Wishart H, Sharpe D. Neuropsychological aspects of multiple sclerosis: a quantitative review. J Clin Exp Neuropsychol 1997;19:810-24.

5 De Sonneville LM, Boringa JB, Reuling IE, et al. Information processing characteristics in subtypes of multiple sclerosis. Neuropsychologia 2002;40:1751-65.
6 Koopmans RA, Li DK, Oger JJ, et al. Chronic progressive multiple sclerosis: serial magnetic resonance brain imaging over six months. Ann Neurol 1989;26:248-56

7 Comi G, Filippi M, Martinelli V, et al. Brain magnetic resonance imaging correlates of cognitive impairment in multiple sclerosis. J Neurol Sci 1993:115:S66-73.

8 Kujala P, Portin R, Ruutiainem J. The progress of cognitive decline in Multiple Sclerosis. A controlled 3-year follow-up. Brain 1997; 120:289-97

9 Rao SM, Hammeke TA, McQuillen MP, et al. Memory disturbance in chronic progressive multiple sclerosis. Arch Neurol 1984;41:625-33.

10 Heaton RK, Nelson LM, Thompson DS, et al. Neuropsychological findings in relapsing-remitting and chronic-progressive multiple sclerosis. J Consult Clin Psychol 1985;53:103-10.

11 Beatty WW, Goodkin DE, Monson N, et al. Cognitive disturbance in patients with relapsing-remitting multiple sclerosis. Arch Neurol 1989:46:1113-19.

12 Hohol MJ, Guttmann CR, Orav J, et al. Serial neuropsychological assessment and magnetic resonance imaging analysis in multiple sclerosis. Arch Neurol 1997;54:1018-25.

13 Comi G, Rovaris M, Falautano M, et al. A multiparametric MRI study of frontal lobe dementia in multiple sclerosis. J Neurol Sci $1999 ; 171: 135-44$

14 Swirsky-Sacchetti T, Mitchell DR, Seward J, et al. Neuropsychological and structural brain lesions in multiple sclerosis: a regional analysis. Neurology 1992:42:1291-95.

15 Jennekens-Schinkel A, Laboyrie PM, Lanser JBK, et al. Cognition in patients with Multiple Sclerosis After for four years. J Neurol Sci 1990;99:229-47

16 Amato MP, Ponziani G, Pracucci G, et al. Cognitive impairment in early-onset Multiple Sclerosis. Pattern, predictors and impact on everyday life in a 4-year follow up. Arch Neurol 1995;52:168-72.

17 Amato MP, Ponziani G, Siracusa G, et al. Cognitive dysfunction in early-onset multiple sclerosis. A reppraisal after 10 years. Arch Neurol 2001;58:1602-6.

18 Mariani C, Farina E, Cappa SF, et al. Neuropsychological assessment in multiple sclerosis: a follow up study with magnetic resonance imaging J Neurol 1991;238:395-400

19 Patti F, Failla G, Ciancio MR, et al. Neuropsychological, neuroradiological and clinical findings in Multiple Sclerosis. A 3-years follow-up study. Eur J Neurol 1998:5:283-6.

20 Kurtzke JF. Rating neurologic imparirment in multiple sclerosis: an expanded disability status scale (EDSS). Neurology 1983;33:1444-52.

21 Feinstein A, Kartsounis LD, Miller DH, et al. Clinically isolated lesions of the type seen in Multiple Sclerosis: a cognitive, psychiatric and MRI follow-up study. J Neurol Neurosurg Psychiatry 1992;55:869-76.

22 Zivadinov R, Sepcic J, Nasuelli D, et al. A longitudinal study of brain atrophy and cognitive disturbances in the early phase of relapsing-remitting multiple sclerosis. J Neurol Neurosurg Psychiatry 2001;70:773-80.

23 Sperling RA, Guttmann CRG, Hohol M, et al. Regional magnetic resonance imaging lesion burden and cognitive function in multiple sclerosis.A longitudinal study. Arch Neurol 2001;58:115-21.

24 Tourtellotte WW, Syndulko K, Jennings P. Use of P300 and a dementia rating scale in the evaluation of cognitive dysfunction in Multiple Sclerosis. Acta Neurol Scand Suppl 1984;101:32-4.

25 Newton MR, Barrett G, Callanan MM, et al. Cognitive event-related potentials in multiple sclerosis. Brain 1989; 112:1637-60.

26 Triantafylliou NI, Zalonis I, Voumvourakis K, et al. Cognition in relapsing-remitting Multiple Sclerosis: a multichannel event-related potential (P300) study. Acta Neurol Scand 1992;85:10-13.

27 Honig LS, Ramsay RE, Sheremata WA. Event-related potentials P300 in multiple sclerosis. Relation to magnetic resonance imaging and cognitive impairment. Arch Neurol 1992;49:44-50.

28 Boose MA, Crandford JL. Auditory event-related in multiple sclerosis. Am J Otol 1996:17:165-70.

29 Sailer M, Heinze HJ, Tendolkar I, et al. Influence of cerebral lesion volume and lesion distribution on event-related potentials in multiple sclerosis. J Neurol 2001;248: 1049-55.

30 Poser CM, Paty DW, Scheinberg L, et al. New diagnostic for multiple sclerosis: guidelines for research protocols. Ann Neurol 1983;13:227-31.

31 Hamilton $\mathbf{M}$. Development of a rating scale for primary depressive illness. Br J Soc Clin Psychol 1967;6:278-96.

32 Basso A, Capitani E, Vignolo P. Esame del linguaggio. Associazione per lo sviluppo delle ricerche Neuropsicologiche. Organizzazioni Speciali Firenze 1974. 1 st edn. Aurora: Stabilimento Grafico, 1987

33 Wechsler D. WAIS Scala di Intelligenza Wechsler per Adulti. San Antonio, TX: The Psychological Corporation, 1955. (Italian version by Ferradini FG, Vassena A. Florence, OS Organizzazioni Speciali 1986).

34 Lezak M. Neuropsychological assesment. Oxford: Oxford University Press, 1983.

35 Wechsler D. WISC-R Scala di Intelligenza Wechsler per Bambini-Riveduta..New York: The Psychological Corporation, 1986. (Italian version by Rubini V, Padovani F. Florence: Organizzazioni Speciali, 1974).

36 Basso A, Capitani E, Laiacona M. Raven's coloured progressive matrices: normative values on 305 adult normal controls. Funct Neurol 1987;2: 189-94.

37 Spinnler H, Tognoni G. Gruppo Italiano per lo Studio Neuropsicologico dell'Invecchiamento: Standardizzazione e taratura italiana di test neuropsicologici. It J Neurol Sci 1987; suppl 8:6. 
38 Buschke $\mathbf{H}$, Fuld PA. Evaluating storage, retention and retrieval in disordered memory and learning. Neurology 1974;24:1019-25.

39 Camp SJ, Stevenson VL, Thompson AJ, et al. Cognitive function in primary progressive and transitional progressive multiple sclerosis. A controlled study with MRI correlates. Brain 1999:122:1341-8.

40 Ormerod IEC, Miller DH, McDonald Wl, et al. The role of NMR imaging in the assessment of multiple sclerosis and isolated neurological lesions. A quantitative study. Brain 1987;110:1579-616.

41 Maurelli M, Marchioni E, Cerretano R, et al. Neuropsychologica assessment in MS: clinical, neurophysiological and neuroradiological relationships. Acta Neurol Scand 1992;86:124-8.

42 Rovaris $M$, lannucci $G$, Falautano $M$, et al. Cognitive dysfunction in patients with midly disabling relapsing-remitting multiple sclerosis: an exploratory study with diffusion tensor MR imaging. J Neurol Sci 2002; 195: 103-9.

43 Faglioni P, Bertolani L, Botti C, et al. Verbal learning strategies in patients with multiple sclerosis. Cortex 2000;36:243-63.

44 Mattioli F, Cappa SF, Cominelli C, et al. Serial study of neuropsychological performance and gadolinium-enhanced MRI in MS. Acta Neurol Scand 1991;87:465-8.

45 Isaac C, Li DBK, Genton M, et al. Multiple sclerosis: a serial study using MRI in relapsing patients. Neurology 1988;38:1511-15.

46 Willoughby EW, Grochowski E, Li DBK, Oger J, et al. Serial magnetic resonance scanning in multiple sclerosis: a second prospective study in relapsing patients. Ann Neurol 1989;25:43-9.

47 Filippi M, Horsfield MA, Tofts PS, et al. Quantitative assessment of MRI lesion load in monitoring the evolution of multiple sclerosis. Brain 1995;118:1601-12.

48 Fulton JC, Grossman RI, Udupa J, et al. MR lesion load and cognitive function in patients with relapsing-remitting multiple sclerosis. ANNR Am J Neuroradiol 1999;20:1951-5.

49 Grimaud J, Barker GJ, Wang L. et al. Correlation of magnetic resonance imaging parameters with clinical disability in multiple sclerosis: a preliminary study. J Neurol 1999;246:961-7.

50 Sinnige LGF, Teeuwissen E, Hew JM, et al. Correlation betwen magnetic resonance imaging and clinical parameters in Multiple Sclerosis. Acta Neurol Scand 1995;91:188-91.

51 Rao SM, Leo GJ, Haughton VM, et al. Correlation of magnetic resonance imaging with neuropsychological testing in multiple sclerosis. Neurology 1989;39:161-6.
52 Arnett PA, Rao SM, Bernardin L, et al. Relationship between frontal lobe lesions and Wisconsin Card Sorting Test performance in patients with multiple sclerosis. Neurology 1994;44:420-5.

53 Foong J, Rozewicz L, Quaghebeur G, et al. Executive function in multiple sclerosis. The role of frontal lobe pathology. Brain 1997; 120:15-26.

54 Rovaris M, Filippi M, Falautano $M$, et al. Relation between MR abnormalities and patterns of cognitive impairment in multiple sclerosis. Neurology 1998;50:1601-8.

55 Moriarty DM, Blackshaw AJ, Talbot PR, et al. Memory dysfunction in multiple sclerosis corresponds to juxtacortical lesion load on fast-attenuated inversion-recovery MR images. Am J Neuroradiol 1999;20: 1956-62

56 Filippi $M$, Tortorella $C$, Rovaris $M$, et al. Changes in the normal appearing brain tissue and cognitive impairment in multiple sclerosis. $J$ Neurol Neurosurg Psychiatry 2000;68:157-61

57 Halgren E, Baudena P, Clarke JM, et al. Intracerebral potentials to rare target and distractor auditory and visual stimuli. I. Superior temporal plane and parietal lobe. Electroencephalogr Clin Neurophysiol 1995a;94:191-220.

58 Halgren $E$, Baudena $\mathrm{P}$, Clarke JM et al.Intracerebral potentials to rare target and distractor auditory and visual stimuli. II. Medial, lateral and posterior temporal lobe. Electroencephalogr Clin Neurophysiol $1995 \mathrm{~b} ; 94: 229-50$.

59 Halgren E, Marinkovic K, Chauvel P. Generators of the late cognitive potentials in auditory and visual oddball tasks. Electroencephalogr Clin Neurophysiol 1998;106:156-64.

60 Linden DE, Prvulovic D, Formisano E, et al. The functional neuroanatomy of target detection: an FMRI study of visual and auditory oddball tasks. Cereb Cortex 1999;9:815-23.

61 Stevens AA, Skudlarski P, Gatenby JC, et al. Event-related FMRI of auditory and visual oddball tasks. Magn Reson Imaging 2000: 18:495-502

62 Polich J, Romine JS, Sipe JC, et al. P300 in multiple sclerosis: a preliminary report. Int J Psychophysiol 1992;12:155-63.

63 Gil R, Zai L, Neau JP, et al. Event-related auditory evoked potentials and multiple sclerosis. Electroencephalogr Clin Neurophysiol 1993:88: 182-7.

64 Comi G, Martinelli V, Locatelli T, et al. Neuropsychological and cognitive markers of disease evolution in Multiple Sclerosis. Mult Scler 1998;4:260-5

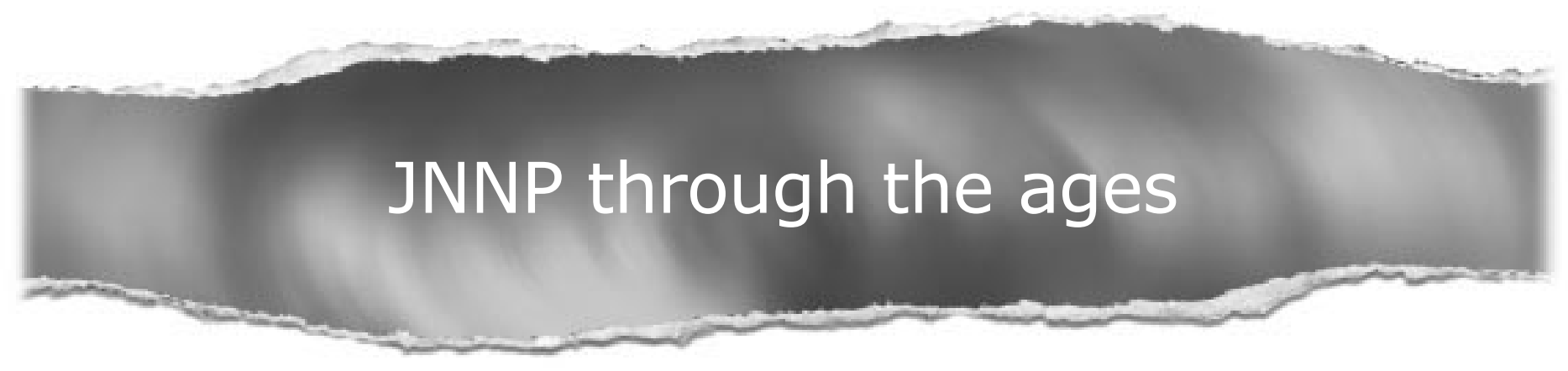

Browse the Archive

Journal of Neurology, Neurosurgery, and Psychiatry online has an archive of content dating back to 1966.

Full text from 1997; abstracts from 1975; table of contents from 1966

www.jnnp.com 